\title{
Working towards full eradication of lipid-driven cardiovascular risk?
}

\author{
N. S. Nurmohamed · E. S. G. Stroes
}

Accepted: 12 May 2021 / Published online: 19 July 2021

(C) The Author(s) 2021

\begin{abstract}
Lipid-driven cardiovascular disease (CVD) risk is caused by atherogenic apolipoprotein B (apoB) particles containing low-density lipoprotein cholesterol (LDL-C), triglycerides and lipoprotein(a) [Lp(a)] and resembles a large and modifiable proportion of the total CVD risk. While a surplus of novel lipid-lowering therapies has been developed in recent years, management of lipid-driven CVD risk in the Netherlands remains suboptimal. To lower LDL-C levels, statins, ezetimibe and proprotein convertase subtilisin/kexin type 9 inhibiting antibodies are the current standard of therapy. With the approval of bempedoic acid and the silencing RNA inclisiran, therapeutic options are expanding continuously. Although the use of triglyceride-lowering therapies remains a matter of debate, post hoc analyses consistently show a benefit in subsets of patients with high triglyceride or low high-density lipoprotein cholesterol levels. Pemafibrate and novel apoC-III could be efficacious options when approved for clinical use. Lp(a)-lowering therapies such as pelacarsen are under clinical investigation, offering a potent $\mathrm{Lp}(\mathrm{a})$-lowering effect. If proven effective in reducing cardiovascular endpoints, $\operatorname{Lp}(\mathrm{a})$ lowering holds promise to be the third axis of effective lipid-lowering therapies. Using these three components of lipid-lowering treatment, the contribution of apoB-containing lipid particles to the CVD risk may be fully eradicated in the next decade.
\end{abstract}

N. S. Nurmohamed · E. S. G. Stroes $(\bowtie)$

Department of Vascular Medicine, Amsterdam UMC,

University of Amsterdam, Amsterdam, The Netherlands e.s.stroes@amsterdamumc.nl

N. S. Nurmohamed

Department of Cardiology, Amsterdam UMC, Vrije

Universiteit, Amsterdam, The Netherlands
Keywords Cardiovascular disease risk - Low-density lipoprotein cholesterol · Lipids · Apolipoprotein · Lipoprotein(a) · Triglycerides

\section{Introduction}

An atherogenic lipid profile is one of the most important risk factors for atherosclerotic cardiovascular disease (CVD) [1]. Lipid-driven risk represents a substantial proportion (approximately 17\%) of the population attributable risk for CVD [2]. First, the largest proportion of the lipid-driven CVD risk is caused by low-density lipoprotein cholesterol (LDL-C) containing apolipoprotein $\mathrm{B}$ (apoB) lipoproteins. About one third of the Western population has dyslipidaemia, defined as an elevation of LDL-C-containing apoB lipoproteins $[1,3]$. Second, in a significant proportion of patients the lipid-driven risk is caused by elevation of triglyceride- and apoB-containing lipoproteins. Almost equal to the prevalence of elevated LDL-C, $31 \%$ of the Western population is hallmarked by a mildto-moderate hypertriglyceridaemia [4]. Third, an estimated $20 \%$ of the world population has elevated lipoprotein(a) [Lp(a)] levels above $50 \mathrm{mg} / \mathrm{dl}$ [5].

While all three risk factors constitute highly prevalent conditions, treatment remains suboptimal and further steps are needed to reduce the impact of lipids on the CVD risk. Thus, $31 \%$ of Dutch secondary prevention and $77 \%$ of primary prevention patients are not achieving their guideline-advised LDL-C targets [6]. Triglyceride-lowering therapies have not been very effective in reducing the CVD risk, while the triglyceride risk burden is increasing due to the pandemic of obesity and diabetes. Finally, approximately $1 \%$ of patients have extremely high $\operatorname{Lp}$ (a) levels exceeding $180 \mathrm{mg} / \mathrm{dl}$, which places them at a familial hypercholesterolaemia (FH)-like risk elevation [7], 
while therapies significantly lowering $\operatorname{Lp}(\mathrm{a})$ levels are not yet in clinical use.

Fortunately, many novel lipid-lowering therapies targeting LDL-C, triglycerides and Lp(a) have emerged over the last decade. The purpose of this review is to discuss current and novel therapies for treating the lipid-driven CVD risk and to support the clinician in the choice between different targets and therapies.

\section{LDL-C/apoB lowering}

Retention and accumulation of apoB-mostly LDLparticles are mandatory for the initiation of atherosclerotic plaque formation. In all major epidemiological studies, the amount of LDL-C and apoB is causally related to the CVD risk. Randomised trials in over 180,000 patients have shown that every $1 \mathrm{mmol} / \mathrm{l}$ reduction of LDL-C is associated with a $19-23 \%$ decrease in CVD risk [8]. Genetic evidence has substantiated that apoB provides the best estimate of the CVD risk [9]. This proves the concept that the CVD risk is determined by the number of apoB particles, rather than the nature of the particle carrying the cholesterol. Mendelian randomisation studies show that lifetime genetic inactivation of pathways targeted by lipid-lowering drugs [3-hydroxy-3-methylglutaryl coenzyme reductase (HMGCR), Niemann-Pick C1like 1 (NPC1L1) and proprotein convertase subtilisinkexin type 9 (PCSK9)] results in comparable relative risk reductions per mmol/l LDL-C or amount of apoB lowering [10, 11]. Taking the duration of exposure into account, an individual's LDL-C-driven CVD risk consists of the cumulative exposure to apoB particles, comparable to the number of pack-years cigarette smoking [12].

\section{Current options: statins, ezetimibe and PCSK9 inhibitors}

The development of the first LDL-C-lowering therapies started in the middle of the past century and led to the development of drugs such as niacin, fibrates and bile acid sequestrants, which did not consistently lower the CVD risk and were hampered by various side effects [13]. Statins were the first LDL-C-lowering therapies to effectively reduce the CVD risk. They lower plasma LDL-C by upregulating hepatic LDL-receptor expression through inhibition of the rate-limiting step of cholesterol biosynthesis, hydroxymethylglutaryl (HMG)-CoA reductase. High-dosed statin therapy leads to a $30-50 \%$ reduction in LDL-C, while less intensive statin regimens yield a $20-30 \%$ LDL-C reduction $[8,14,15]$. Over the years, statins have proven to be very safe. Although adverse effects are relatively common in open-label surveys, randomised controlled trials show a low adverse event rate [16]. Observed adverse effects include new-onset diabetes (10-20 cases per 10,000 patient-years) and myopathy (1 case per 10,000 patient-years) [16].
However, the clinical prevalence of statin intolerance remains high and $7-29 \%$ of patients experience statinassociated muscle symptoms, resulting in a major drawback in statin prescription and adherence [17].

In 2015, the IMPROVE-IT trial provided evidence that ezetimibe was also effective in reducing major adverse cardiovascular events (MACE) when given on top of statins [hazard ratio (HR) $0.94,95 \%$ confidence interval (CI) 0.89-0.99, $p=0.016$ ] after an acute coronary syndrome [18]. Ezetimibe reduced LDL-C by an additional $18-22 \%$ by targeting the NPC1L1 protein, thereby reducing the intestinal absorption of cholesterol $[19,20]$. The results of IMPROVE-IT were confirmed by Mendelian evidence showing that reduction of the CVD risk is comparable per mmol/l genetic LDL-C lowering by NPC1L1 and HMGCR polymorphisms [11]. In the IMPROVE-IT trial, ezetimibe was generally well tolerated and without major adverse effects. However, ezetimibe may result in (albeit mostly mild) gastrointestinal side effects.

PCSK9 inhibitors prevent extracellular binding and degradation of LDL receptors by inhibition of the PCSK9 protein and are the most recent addition to the lipid-lowering armamentarium. Currently available PCSK9 inhibitors are monoclonal antibodies which are administered subcutaneously twice monthly: evolocumab and alirocumab. They were proven effective in the FOURIER and ODYSSEY OUTCOMES trials, respectively, comprising a total of 46,488 patients, showing an additional reduction of $59-62 \%$ in LDL-C when given in addition to statin therapy [21, 22]. Both studies led to an additional 15\% MACE reduction after a mean follow-up of 2.2 and 2.8 years respectively. There were no major safety issues with either drug, and adverse event rates were similar to those with placebo, except for a higher occurrence of injection site reactions with PCKS9 inhibitors. In addition, treatment with PCSK9 inhibitors can result in flu-like symptoms in a small proportion of patients.

\section{Bempedoic acid}

In 2020, both the European Medicines Agency (EMA) and the United States Food and Drug Association (FDA) approved bempedoic acid for use in patients with statin intolerance or patients not reaching LDL-C targets using statin therapy [23, 24]. Bempedoic acid can be prescribed as a daily oral drug and is metabolised in the liver to a coenzyme A (CoA) thioester form, which targets adenosine triphosphate citrate lyase (ACL) upstream of HMG-CoA reductase, resulting in upregulation of LDL receptors and a reduction of plasma LDL-C [25]. Its mechanism was supported by a Mendelian randomisation study in 654,783 subjects, showing variants in the ACL gene, $A C L Y$, resulted in a similar CVD risk reduction per mmol/l LDL-C lowering compared to variants in the statin gene, HMGCR [26]. In clinical trials, bempedoic acid resulted in a $17-23 \%$ reduction of LDL-C when 


\section{Advertisement placed here.}

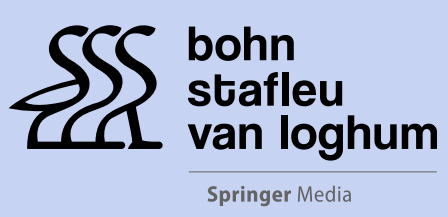

Houten 2021 


\section{Advertisement placed here.}

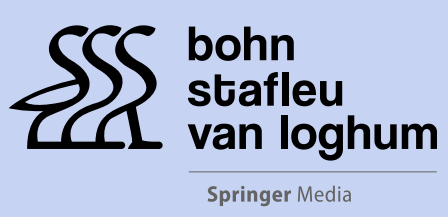

Houten 2021 
given on top of maximally tolerated statin therapy or in statin-intolerant patients [27-29]. Importantly, the incidence of muscle-related adverse events was comparable to that of placebo in these trials. Bempedoic acid has an acceptable safety profile, however did result in an increase of blood uric acid levels and an increased risk of gout $(1.2 \%$ vs $0.3 \%, p=0.03)$ in the phase 3 CLEAR Harmony trial (NCT02666664). The larger phase 3 CLEAR Outcomes trial $(n=14,014)$ will provide a more detailed profile for CV efficacy and safety (NCT02993406).

\section{Inhibition of PCSK9: inclisiran}

In addition to PCSK9-inhibiting monoclonal antibodies, other promising therapies targeting PCSK9 have emerged. Inclisiran is a subcutaneously administered small interfering RNA (siRNA) [30], providing intracellular, hepatocyte-specific PCSK9 inhibition by silencing PCSK9 messenger RNA (mRNA) translation [31]. Inclisiran is long-acting following incorporation into the RNA-induced silencing complex leading to sustained LDL-C reductions, which allows twiceyearly administration. Phase 3 trials have corroborated a consistent $50 \%$ reduction of LDL-C in CVD patients, CVD risk equivalent patients and $\mathrm{FH}$ patients $[32,33]$. Adverse event rates were generally similar between groups, with the exception of (mild) injectionsite reactions occurring more frequently in the treatment groups. The ORION-4 trial will provide longterm data on CV efficacy and safety (NCT03705234). On 11 December 2020, inclisiran was approved by the EMA for the treatment of adults with hypercholesterolaemia or mixed dyslipidaemia, with two doses a year.

\section{ANGPTL3 inhibition}

The liver-expressed, secreted protein angiopoietinlike protein 3 (ANGPTL3) inhibits lipoprotein lipase (LPL), preventing hydrolysis of triglycerides in the peripheral capillaries. In addition, ANGPTL3 also lowers apoB and LDL-C by increasing clearance of LDL particles and reducing hepatic very-low-density lipoprotein (VLDL) secretion [34, 35]. Importantly, its mechanisms of action are independent of the low-density lipoprotein receptor (LDLR) pathway. Genetic studies have shown that ANGPTL3 lossof-function mutations result in a hypobetalipoproteinaemia phenotype, hallmarked by decreased levels of triglycerides, LDL-C and high-density lipoprotein cholesterol (HDL-C) [34, 36]. Inhibition of ANGPTL3 with evinacumab was recently shown to be effective in reducing LDL-C levels by $49 \%$ in a phase 3 trial with $65 \mathrm{FH}$ patients already maximally treated with lipid-lowering therapies [37]. In this study, triglycerides were lowered by $50 \%$ and another phase 3 trial will evaluate its efficacy in patients with severe hypertriglyceridaemia (NCT03452228). There have been no major safety issues in the clinical studies with evinacumab to date, and the FDA has designated evinacumab a breakthrough status, paving the way for efficient approval.

In addition to evinacumab, multiple other drugs targeting ANGPTL3 are currently in clinical trials. IONIS-ANGPTL3-L $\mathrm{L}_{\mathrm{Rx}}$, an antisense oligonucleotide (ASO) targeting ANGPTL3, showed dose-dependent LDL-C and triglyceride reductions in its phase 1 study [38]. Its phase 2 study is currently underway in patients with type 2 diabetes, hypertriglyceridaemia and fatty liver disease (NCT03371355). ARO-ANG3 is an siRNA agent targeting ANGPTL3, and preliminary data also showed moderate dose-dependent reductions in LDL-C and triglycerides.

\section{Clinical use of LDL-C-lowering agents}

In all patients with a high CVD risk and LDL-C levels above their target according to the European Society of Cardiology (ESC)/European Atherosclerosis Society (ESA) guidelines [7], potent statin therapy is the firstchoice treatment. In all patients not achieving target LDL-C levels, ezetimibe should be added to potent statin therapy. If elevated LDL-C levels are present in a very-high-risk patient, direct initiation of combination therapy of potent statin and ezetimibe can be considered. In cases of very high CVD risk and residual LDL-C burden, PCSK9 inhibition can be considered to further reduce LDL-C. Due to the higher costs of PCSK9-inhibiting antibodies, reimbursement is limited to patients following myocardial infarction and (1) recurrent myocardial infarction; (2) diabetes mellitus; (3) documented statin intolerance; or (4) in patients with $\mathrm{FH}$ not reaching LDL-C target levels despite statin/ezetimibe combination therapy. Pending reimbursement, bempedoic acid can also be considered to reduce LDL-C in patients not reaching the LDL-C target or in patients with statin intolerance. Finally, evinacumab is a promising additional option for patients with (homozygous) FH, since it lowers LDL-C independently of the LDL receptor pathway.

\section{Triglyceride lowering}

Hypertriglyceridaemia is present in approximately $30 \%$ of the Western population, but its burden continues to grow due to the increasing prevalence of obesity and diabetes. Obesity is associated with an atherogenic lipid profile hallmarked by high plasma triglyceride levels [39]. Both population and genetic studies have provided evidence for a causal relationship between triglycerides and CVD [40]. By means of Mendelian randomisation it was shown that triglyceride-lowering variants in the $L P L$ gene achieved a CVD risk reduction equal to that of LDL-C-lowering variants in the $L D L R$ gene per unit of apoB lowering [41]. This supports the concept that the CVD risk reduction is dependent on the reduction of apoB 
particles, and not on the magnitude of LDL-C or triglyceride lowering.

\section{Current options: fibrates and omega-3 fatty acids}

The dependency on apoB reduction may partly explain the failure of large fibrate trials to show a solid reduction in CVD, since the achieved apoB lowering using fibrate therapy is minimal [42]. However, in post hoc analyses, fibrates were found to reduce the CVD risk in patients with elevated triglyceride and/or low HDL-C levels at baseline [43-45]. Pemafibrate is a novel fibrate which selectively targets peroxisome proliferator-activated receptor (PPAR)-alpha. Given orally once daily, pemafibrate appears to have a favourable adverse effect profile and is more potent, offering both LDL-C as well as apoB lowering [46, 47]. The ongoing PROMINENT trial evaluating pemafibrate in more than 10,000 type 2 diabetes patients with mixed dyslipidaemia will show whether there is a place for triglyceride-lowering regimens in future CVD prevention (NCT03071692).

Another point of debate is the use of omega-3 fatty acids. The supposed mechanism of action of daily dosed omega-3 fatty acids is largely unknown and pleiotropic [48]. After failure of almost all omega-3 fatty acid trials, REDUCE-IT was the first study to show a significant MACE reduction [49]. In 8179 patients, high-dose eicosapentaenoic acid (EPA) significantly reduced the primary endpoint in the intervention group compared to placebo (HR 0.75, 95\% CI $0.68-0.83, p<0.001)$. More recently, the STRENGTH trial in 13,078 patients, which evaluated high-dose EPA in combination with docosahexaenoic acid, was terminated early due to its futility [50]. Whether the discrepancy between these studies in part reflects the poorly chosen placebo (mineral oil) in the REDUCEIT trial or is the result of differences between the two formulations remains a matter of debate. Importantly, the CVD benefit in the REDUCE-IT trial is unlikely to be solely attributable to the modest triglyceride lowering by high-dose EPA [48].

\section{ANGPTL3 inhibition}

For patients with a residual triglyceride risk, or a combined LDL-C/triglyceride risk, inhibition of ANGPTL3 is an interesting therapeutic option that lowers both LDL-C and triglycerides. It is discussed in the LDL-C/apoB-lowering section.

\section{Targeting of apoC-III}

ApoC-III (apoC-III) is an apolipoprotein mainly carried by triglyceride-rich lipoproteins, such as chylomicrons and VLDLs. apoC-III prevents hydrolysis of triglycerides by LPL, for example [51]. In a genetic study, carriers of loss-of-function mutations in APOC3 had an HR of 0.59 (95\% CI $0.41-0.86, p=0.007$ ) and
0.64 (95\% CI 0.41-0.99, $p=0.04$ ) for ischaemic vascular disease and ischaemic heart disease respectively [52]. Volanesorsen, an ASO, was the first apoC-III inhibitor reaching clinical trials, and is given as a subcutaneous formulation twice monthly. Treatment with volanesorsen effectively reduces plasma triglyceride levels in familial chylomicronaemia syndrome and hypertriglyceridaemia by approximately $70-80 \%$ [53]. However, volanesorsen is hampered by the high incidence of adverse effects, including thrombocytopenia and injection-site reactions [54], precluding use in large patient populations. Recently, a N-acetyl galactosamine-conjugated (GalNAc ${ }_{3}$ ) ASO targeting apoCIII (AKCEA-APOCIII- $\mathrm{L}_{\mathrm{Rx}}$ ) was tested in a phase $1 / 2 \mathrm{a}$ trial [55]. The GalNAc ${ }_{3}$ formulation enables specific delivery to the liver, increasing potency while minimising systemic exposure. Indeed, the trial showed potent reductions in triglycerides up to $92 \%$, and administration was without major adverse effects.

\section{Clinical use of triglyceride-lowering agents}

Since the CVD benefit of current triglyceride-lowering agents is controversial, they should not be prescribed routinely. Fibrates can be considered only in veryhigh-risk patients with persisting high triglyceride levels whose risk cannot be reduced with LDL-C lowering. EPA can also be considered in patients with a high CVD risk who have elevated triglyceride levels, although EPA has not been approved by the EMA to date. Highly potent pemafibrate, if shown to be effective and tolerable, could prove an interesting option in patients with an atherogenic lipid profile with high triglyceride levels, such as diabetes patients. AKCEAAPOCIII- $\mathrm{L}_{\mathrm{Rx}}$, the GalNAc $_{3}$ ASO targeting apoC-III, is a promising new therapy to reduce triglycerides and could, if reasonably priced, be a future therapy choice in the patient with a high CVD risk and high triglyceride levels. In patients with a combined 'refractory' LDL-C/triglyceride risk, ANGPTL3 inhibition could kill two birds with one stone.

\section{Lowering of $\operatorname{Lp}(\mathrm{a})$}

$\mathrm{Lp}(\mathrm{a})$ is an LDL-like particle with its apoB covalently bound to an apo(a) tail. Plasma levels are genetically determined for more than $90 \%$ [56]. Elevated plasma $\mathrm{Lp}$ (a) has been established as a causal risk factor for both CVD and calcific aortic valve disease in population and genome-wide association studies [57, 58]. Further genetic evidence shows that gain-of-function mutations in the LPA gene increase the CVD risk, while loss-of-function mutations lead to a reduction of the CVD risk [59]. The prevalence of elevated Lp(a) is high; 1.4 billion people worldwide have an $\mathrm{Lp}$ (a) level above the threshold of $50 \mathrm{mg} / \mathrm{dl}$ [5]. In addition, approximately $1 \%$ have an $\mathrm{Lp}$ (a) level above $180 \mathrm{mg} / \mathrm{dl}$, which puts them at an FH-like risk of CVD [7]. Unfortunately, a large proportion of patients remain uniden- 


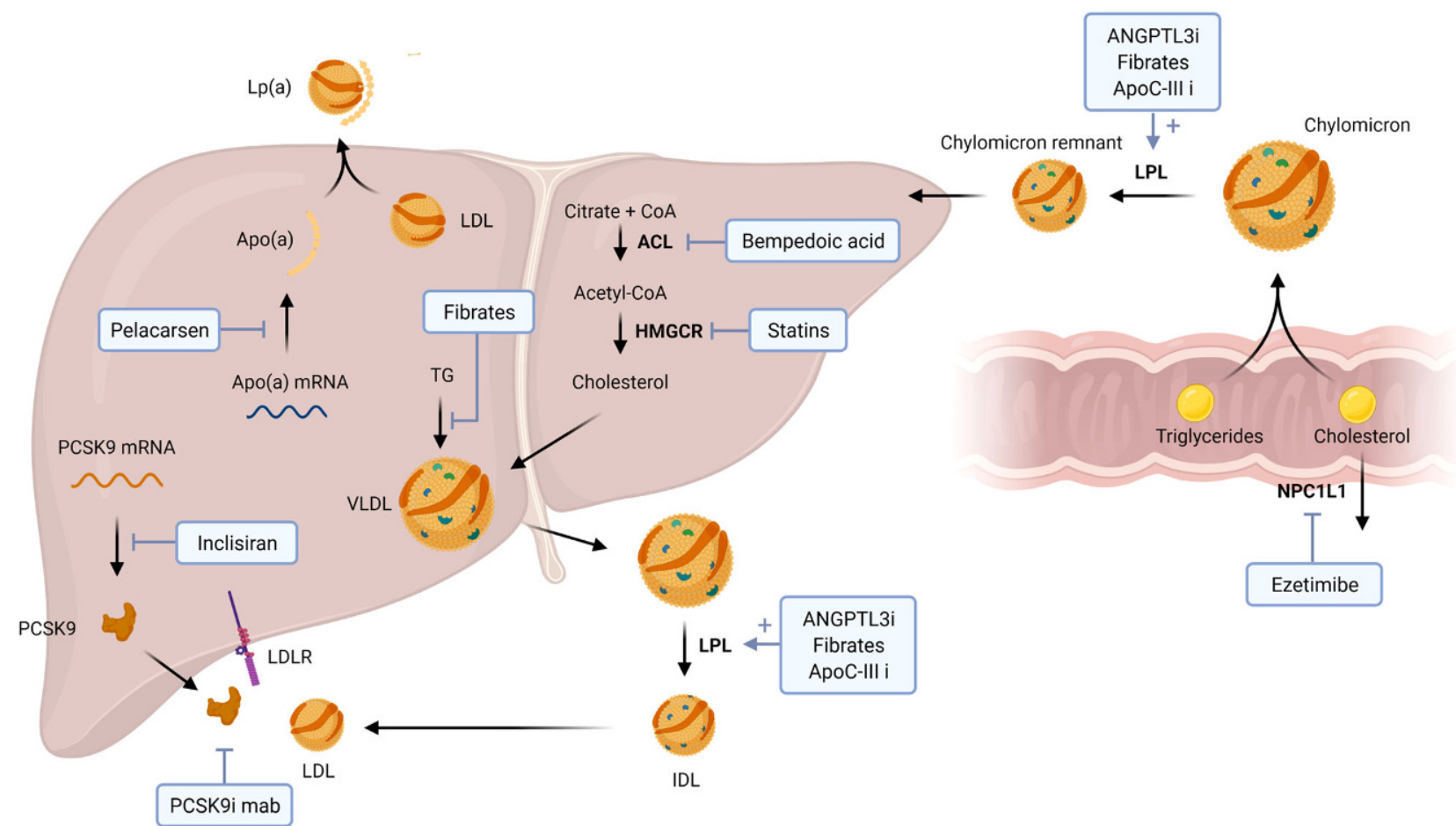

Fig. 1 Mechanisms of action of the discussed lipid-lowering therapies. Pelacarsen inhibits apolipoprotein(a) (apo[a]). Inclisiran prevents translation of proprotein convertase subtilisin kexin type 9 (PCSK9) mRNA. PCSK9i (inhibiting) monoclonal antibodies (mab) inhibit PCSK9 binding to low-density lipoprotein receptor $(L D L R)$. Fibrates mainly prevent synthesis of triglycerides (TG) and very-low-density lipoprotein (VLDL) production in the liver. Bempedoic acid prevents cholesterol synthesis by inhibition of adenosine triphosphate

tified due to unawareness among physicians and the previous lack of an effective Lp(a)-lowering treatment. The latter is about to change: multiple Lp(a)-lowering therapies are currently in clinical trials.

\section{Current options: PCSK9 inhibitors}

Current options to lower $\operatorname{Lp}(\mathrm{a})$ are scarce. Conversely, statins are known to slightly increase $\mathrm{Lp}$ (a) levels up to $10-20 \%$ [60]. The only therapy in use which is known to lower Lp(a) is PCSK9 inhibition. In addition to their effective LDL-C reductions, PCSK9 inhibitors reduce Lp(a) levels by a median of $21-26 \%$ [61]. It was estimated, based on Mendelian randomisation studies, that relatively large absolute $\mathrm{Lp}$ (a) reductions between 50 and $100 \mathrm{mg} / \mathrm{dl}$ are necessary to achieve a CVD risk reduction equal to that of $1 \mathrm{mmol} / \mathrm{l}$ LDL-C reduction [62, 63]. However, a recent analysis of the ODYSSEY OUTCOMES trial has shown that PCSK9 therapy could be of use in patients with very high Lp(a) levels. It was observed that $\mathrm{Lp}$ (a) reduction independently lowered the CVD risk (2.5\% risk reduction per $5 \mathrm{mg} / \mathrm{dl} \mathrm{Lp}$ (a) lowering by alirocumab). The CVD risk reduction achieved with alirocumab in patients in the highest $\operatorname{Lp}$ (a) quartile was for $39 \%$ attributed to $\mathrm{Lp}$ (a) reduction. This suggests that a sub- citrate lyase $(A C L)$. Statins block 3-hydroxy-3-methylglutaryl coenzyme reductase (HMGCR). Angiopoetin-like 3 protein inhibitors (ANGPTL3i), fibrates and apoC-III inhibitors (apoC-IIIi) enhance lipoprotein lipase $(L P L)$ function. Ezetimibe targets Niemann-Pick C1-like 1 protein (NPC1L1), inhibiting transport of sterols into enterocytes. Created with BioRender (BioRender.com). CoA coenzyme A, IDL intermediate-density lipoprotein, $L p($ a) lipoprotein(a)

group of patients with a high CVD risk and a high Lp(a) level could benefit from PCSK9 inhibition.

\section{Pelacarsen (TQJ230)}

Pelacarsen (ASO) is the first Lp(a)-lowering drug in a phase 3 trial and is administered as a subcutaneous injection once monthly. With its $\mathrm{GalNAc}_{3}$ conjugation, potency is increased while systemic side effects are reduced. Pelacarsen has shown dose-dependent decreases in $\operatorname{Lp}(\mathrm{a})$ up to $92 \%$ in its phase 2 trials [64, 65]. In these trials, there were no major safety issues and pelacarsen was generally well tolerated, with the exception of more injection-site reactions in the pelacarsen group than with placebo. The phase 3 trial of pelacarsen, $\mathrm{Lp}$ (a) HORIZON, is currently recruiting 7680 patients with established CVD and is expected to be completed in 2024 (NCT04023552).

\section{siRNA: AMG890 and SLN360}

Two investigational siRNA therapies targeting Lp(a) mRNA are currently being clinically evaluated. First, AMG890 (olpasiran) is an siRNA,the phase 1 study of which has recently finished, showing dose-dependent reductions of more than $90 \%$ without any safety con- 
cerns [66]. These reductions persisted for 3-6 months in the highest doses. Its phase 2 trial in patients with elevated Lp(a) is currently underway (NCT04270760). Second, SLN360 is another siRNA drug which has shown potent $L p(a)$ reduction without any adverse or off-target effects in animal studies;its phase 1 study will be starting soon (NCT04606602).

\section{Clinical use of Lp(a)-lowering agents}

If proven effective for lowering of the CVD risk, and approved by regulatory agencies, Lp(a)-lowering treatments could provide the clinician with the last tool to abolish residual apoB-mediated lipid risk. These therapies should be prescribed in patients with a high CVD risk (secondary prevention) with elevated $\operatorname{Lp}(\mathrm{a})$ levels $(>50 \mathrm{mg} / \mathrm{dl})$. In patients with extremely high levels $>180 \mathrm{mg} / \mathrm{dl}$ and hence a markedly increased lifetime CVD risk, Lp(a) lowering should also be considered. Following the most recent ESC/EAS dyslipidaemia guidelines, every at-risk individual should have their $\operatorname{Lp}$ (a) levels measured at least once in their lifetime. It is especially important to measure $\operatorname{Lp}(\mathrm{a})$ when measuring other lipids, since Lp(a) cholesterol and $\mathrm{Lp}(\mathrm{a})-\mathrm{apoB}$ are measured in the total concentration of LDL-C or apoB respectively. Therefore, in patients with very high $\mathrm{Lp}$ (a) levels, the measured LDL-C and apoB could be 'falsely' elevated and actually reflect elevated $\mathrm{Lp}(\mathrm{a})-\mathrm{C}$ and $\mathrm{Lp}(\mathrm{a})$-apoB. These patients would benefit much more from $\mathrm{Lp}$ (a) lowering than from LDL-C/apoB lowering. This problem could be solved by routinely reporting LDL-C levels corrected for $\mathrm{Lp}(\mathrm{a})-\mathrm{C}$ [67].

\section{Conclusions}

There is an abundance of lipid-lowering therapies under development in addition to the currently available regimens (Fig. 1). The vast majority of these agents are safe with minimal side effects, which compares favourably to agents targeting other pathways such as coagulation (bleeding risk) and inflammation (infection risk). In each individual patient, a personalised treatment dependent on their CVD risk and respective lipid profile should be configured. In patients with a high LDL-C risk, bempedoic acid and the twice-yearly dosed inclisiran are novel options on top of guideline-advised statins, ezetimibe and PCSK9-inhibiting antibodies. When high triglyceride levels are the main cause of the residual CVD risk, options to lower this risk are currently limited, but pemafibrate and novel apoC-III inhibitors provide a future perspective. For patients with high Lp(a) levels, pelacarsen is the first of several therapies which potently lower $\mathrm{Lp}(\mathrm{a})$, and can be prescribed if proven safe and effective in reducing MACE. In summary, the contribution of apoB-containing lipid particles to the CVD risk can be fully eradicated within the next decade in a safe and highly effective manner.
Conflict of interest N.S. Nurmohamed is co-founder of Lipid Tools. E.S.G. Stroes reports advisory board/lecturing fees paid to his institution by Amgen, Sanofi-Regeneron, Mylan, Esperion, Novartis, as well as grants from Athera, all outside the scope of the submitted work.

Open Access This article is licensed under a Creative Commons Attribution 4.0 International License, which permits use, sharing, adaptation, distribution and reproduction in any medium or format, as long as you give appropriate credit to the original author(s) and the source, provide a link to the Creative Commons licence, and indicate if changes were made. The images or other third party material in this article are included in the article's Creative Commons licence, unless indicated otherwise in a credit line to the material. If material is not included in the article's Creative Commons licence and your intended use is not permitted by statutory regulation or exceeds the permitted use, you will need to obtain permission directly from the copyright holder. To view a copy of this licence, visit http://creativecommons.org/licenses/by/4.0/.

\section{References}

1. Borén J, Chapman MJ, Krauss RM, et al. Low-density lipoproteins cause atherosclerotic cardiovascular disease: pathophysiological, genetic, and therapeuticinsights: a consensus statement from the European atherosclerosis society consensus panel. Eur Heart J. 2020;41:2313-30.

2. Pencina MJ, Navar AM, Wojdyla D, et al. Quantifying importance of major risk factors for coronary heart disease. Circulation. 2019;139:1603-11.

3. GoffDCJr, Bertoni AG, Kramer H, et al. Dyslipidemia prevalence, treatment, and control in the multi-ethnic study of atherosclerosis (MESA). Circulation. 2006;113:647-56.

4. Miller M, Stone NJ, Ballantyne C, et al. Triglycerides and cardiovascular disease. Circulation. 2011;123:2292-333.

5. Tsimikas S, Stroes ESG. The dedicated "Lp(a) clinic": a conceptwhosetimehas arrived? Atherosclerosis. 2020;300:1-9.

6. Balder JW, Scholtens S, de Vries JK, et al. Adherence to guidelines to prevent cardiovascular diseases: the lifelines cohortstudy. Neth J Med. 2015;73:316-23.

7. Mach F, Baigent C, Catapano AL, et al. 2019 ESC/EAS guidelines for the management of dyslipidaemias: lipid modification to reduce cardiovascular risk. Eur Heart J. 2020;4:111-88.

8. Armitage J, Baigent C, Barnes E, et al. Efficacy and safety of statin therapy in older people: a meta-analysis of individual participant data from 28 randomised controlled trials. Lancet. 2019;393:407-15.

9. Ference BA, Kastelein JJP, Catapano AL. Lipids and lipoproteins in 2020. JAMA. 2020;324:595-6.

10. Ference BA, Robinson JG, Brook RD, et al. Variation in PCSK9 and HMGCR and risk of cardiovascular disease and diabetes. NEngl J Med. 2016;375:2144-53.

11. Ference BA, Majeed F, Penumetcha R, Flack JM, Brook RD. Effect of naturally random allocation to lower low-density lipoprotein cholesterol on the risk of coronary heart disease mediated by polymorphisms in NPC1L1, HMGCR, or both: a $2 \times 2$ factorial mendelian randomization study. J Am Coll Cardiol. 2015;65:1552-61.

12. Ference BA, Graham I, Tokgozoglu L, Catapano AL. Impact of lipids on cardiovascular health: JACC health promotion series. JAm Coll Cardiol. 2018;72:1141-56.

13. Pedersen TR. The success story of LDLcholesterollowering. Circ Res. 2016;118:721-31.

14. Baigent C, Blackwell L, Emberson J, et al. Efficacy and safety of more intensive lowering of LDL cholesterol: a meta- 
analysis of data from 170000 participants in 26 randomised trials. Lancet. 2010;376:1670-81.

15. Fulcher J, O'Connell R, Voysey M, et al. Efficacy and safety of LDL-lowering therapy among men and women: metaanalysis of individual data from 174000 participants in 27 randomised trials. Lancet. 2015;385:1397-405.

16. Collins R, Reith C, Emberson J, et al. Interpretation of the evidence for the efficacy and safety of statin therapy. Lancet. 2016;388:2532-61.

17. Stroes ES, Thompson PD, Corsini A, et al. Statin-associated muscle symptoms: impact on statin therapy-European atherosclerosis society consensus panel statement on assessment, aetiology and management. Eur Heart J. 2015;36:1012-22.

18. Cannon CP, Blazing MA, Giugliano RP, et al. Ezetimibe added to statin therapy after acute coronary syndromes. NEngl J Med. 2015;372:2387-97.

19. Sudhop T, Lütjohann D, Kodal A, et al. Inhibition of intestinal cholesterol absorption by ezetimibe in humans. Circulation. 2002;106:1943-8.

20. Morrone D, Weintraub WS, Toth PP, et al. Lipid-altering efficacy of ezetimibe plus statin and statin monotherapy and identification of factors associated with treatment response: a pooled analysis of over 21,000 subjects from 27 clinical trials. Atherosclerosis. 2012;223:251-61.

21. Sabatine MS, Giugliano RP, KeechAC, etal. Evolocumab and clinical outcomes in patients with cardiovascular disease. NEngl J Med. 2017;376:1713-22.

22. Schwartz GG, Steg PG, Szarek M, et al. Alirocumab and cardiovascular outcomes after acute coronary syndrome. NEngl J Med. 2018;379:2097-107.

23. European Medicines Agency. Nilemdo. 2020. https://www. ema.europa.eu/en/medicines/human/EPAR/nilemdo. Accessed 9 Oct 2020.

24. Food and Drug Administration. Drug approval package: NEXLETOL. 2020. https://www.accessdata.fda.gov/ drugsatfda_docs/nda/2020/211616Origls000TOC.cfm. Accessed 27 July 2020.

25. Pinkosky SL, Newton RS, Day EA, et al. Liver-specific ATP-citrate lyase inhibition by bempedoic acid decreases LDL-C and attenuates atherosclerosis. Nat Commun. 2016;7:13457.

26. Ference BA, Ray KK, CatapanoAL, etal. Mendelian randomization study of ACLY and cardiovascular disease. N Engl J Med. 2019;380:1033-42.

27. Ray KK, Bays HE, Catapano AL, et al. Safety and efficacy of bempedoic acid to reduce LDL cholesterol. N Engl J Med. 2019;380:1022-32.

28. Goldberg AC, Leiter LA, Stroes ESG, et al. Effect of bempedoic acid vs placebo added to maximally tolerated statins on low-density lipoprotein cholesterol in patients at high risk for cardiovascular disease: the CLEAR wisdom randomized clinical trial. JAMA. 2019;322:1780-8.

29. Laufs U, Banach M, Mancini GBJ, etal. Efficacy and safety of bempedoic acid in patients with hypercholesterolemia and statin intolerance. JAm HeartAssoc. 2019;8:e11662.

30. Fitzgerald K, Frank-Kamenetsky M, Shulga-Morskaya S, et al. Effect of an RNA interference drug on the synthesis of proprotein convertase subtilisin/kexin type 9 (PCSK9) and the concentration of serum LDL cholesterol in healthy volunteers: a randomised, single-blind, placebo-controlled, phase 1 trial. Lancet. 2014;383:60-8.

31. Fitzgerald K, White S, Borodovsky A, et al. A highly durable RNAi therapeutic inhibitor of PCSK9. N Engl J Med. 2017;376:41-51.
32. Raal FJ, Kallend D, Ray KK, et al. Inclisiran for the treatment of heterozygous familial hypercholesterolemia. N Engl J Med. 2020;382:1520-30.

33. Ray KK, Wright RS, Kallend D, et al. Two phase 3 trials of inclisiran in patients with elevated LDL cholesterol. NEngl J Med. 2020;382:1507-19.

34. Musunuru K, Pirruccello JP, Do R, et al. Exome sequencing, ANGPTL3 mutations, and familial combined hypolipidemia. NEngl J Med. 2010;363:2220-7.

35. Wang Y, Gusarova V, Banfi S, Gromada J, Cohen JC, Hobbs HH. Inactivation of ANGPTL3 reduces hepatic VLDL-triglyceride secretion. J Lipid Res. 2015;56:1296-307.

36. Stitziel NO, Khera AV, Wang X, et al. ANGPTL3 deficiency and protection against coronary artery disease. J Am Coll Cardiol. 2017;69:2054-63.

37. Raal FJ, Rosenson RS, Reeskamp LF, et al. Evinacumab for homozygous familial hypercholesterolemia. N Engl J Med. 2020;383:711-20.

38. Graham MJ, Lee RG, Brandt TA, et al. Cardiovascular and metabolic effects of ANGPTL3 antisense oligonucleotides. NEnglJ Med. 2017;377:222-32.

39. Björnson E, Adiels M, Taskinen M-R, Borén J. Kinetics of plasma triglycerides in abdominal obesity. Curr Opin Lipidol. 2017;28:11-8.

40. Triglyceride Coronary Disease Genetics Consortium and Emerging Risk Factors Collaboration, et al. Triglyceridemediated pathways and coronary disease: collaborative analysis of 101 studies. Lancet. 2010;375:1634-9.

41. Ference BA, Kastelein JJP, Ray KK, et al. Association of triglyceride-lowering LPL variants and LDL-C-lowering LDLR variants with risk of coronary heart disease. JAMA. 2019;321:364-73.

42. Barter PJ, Rye K-A. New era of lipid-lowering drugs. Pharmacol Rev. 2016;68:458-75.

43. Scott R, O'Brien R, Fulcher G, et al. Effects of fenofibrate treatment on cardiovascular disease risk in 9,795 individuals with type 2 diabetes and various components of the metabolic syndrome. Diabetes Care. 2009;32:493-8.

44. Behar S, Brunner D, Kaplinsky E, Mandelzweig L, Benderly M. Secondary prevention by raising HDL cholesterol and reducing triglycerides in patients with coronary artery disease: the bezafibrate infarction prevention (BIP) study. Circulation. 2000;102:21-7.

45. Manninen V, Tenkanen L, Koskinen P, et al. Joint effects of serum triglyceride and LDL cholesterol and HDL cholesterol concentrations on coronary heart disease risk in the Helsinki heart study. Implications for treatment. Circulation. 1992;85:37-45.

46. Ishibashi S, Arai H, Yokote K, Araki E, Suganami H, Yamashita S. Efficacy and safety of pemafibrate (K-877), a selective peroxisome proliferator-activated receptor $\alpha$ modulator, in patients with dyslipidemia: results from a 24week, randomized, doubleblind, active-controlled, phase 3 trial. JClin Lipidol. 2018;12:173-84.

47. Araki E, Yamashita S, Arai H, et al. Effects of pemafibrate, a novel selective PPAR $\alpha$ modulator, on lipid and glucose metabolism in patients with type 2 diabetes and hypertriglyceridemia: a randomized, double-blind, placebocontrolled, phase 3 trial. Diabetes Care. 2018;41:538-46.

48. Kastelein JJP, Stroes ESG. FISHing for the miracle of eicosapentaenoic acid. NEngl J Med. 2018;380:89-90.

49. Bhatt DL, Steg PG, Miller M, et al. Cardiovascular risk reduction with icosapent ethyl for hypertriglyceridemia. NEngl J Med. 2018;380:11-22.

50. Nicholls SJ, Lincoff AM, Garcia M, et al. Effect of highdose omega-3 fatty acids vs corn oil on major adverse cardiovascular events in patients at high cardiovascular 
risk: the STRENGTH randomized clinical trial. JAMA. 2020;324:2268-80.

51. Ginsberg HN, Le NA, Goldberg IJ, et al. Apolipoprotein B metabolism in subjects with deficiency of apolipoproteins CIII and AI. Evidence that apolipoprotein CIII inhibits catabolism of triglyceride-rich lipoproteins by lipoprotein lipase in vivo. JClin Invest. 1986;78:1287-95.

52. Jørgensen AB, Frikke-Schmidt R, Nordestgaard BG, Tybjærg-Hansen A. Loss-of-function mutations in APOC3 and risk of ischemic vascular disease. N Engl J Med. 2014;371:32-41.

53. WitztumJL, GaudetD, FreedmanSD, etal. Volanesorsen and triglyceride levels in familial chylomicronemia syndrome. NEngl J Med. 2019;381:531-42.

54. Khetarpal SA, et al. Volanesorsen, familial chylomicronemia syndrome, and thrombocytopenia. N Engl J Med. 2019;381:2582-4.

55. Alexander VJ, Xia S, Hurh E, et al. N-acetyl galactosamine-conjugated antisense drug to APOC3 mRNA, triglycerides and atherogeniclipoproteinlevels. EurHeartJ. 2019;40:2785-96.

56. Sang-Rok L, Anand P, Yun-Seok C, et al. LPA Gene, ethnicity, and cardiovascular events. Circulation. 2017;135:251-63.

57. Clarke R, Peden JF, Hopewell JC, et al. Genetic variants associated with Lp(a) lipoprotein level and coronary disease. NEngl J Med. 2009;361:2518-28.

58. Thanassoulis G, Campbell CY, Owens DS, et al. Genetic associations with valvular calcification and aortic stenosis. NEngl J Med. 2013;368:503-12.

59. Emdin CA, Khera AV, Natarajan P, et al. Phenotypic characterization of genetically lowered human lipoprotein(a) levels. JAm Coll Cardiol. 2016;68:2761-72.

60. Tsimikas S, Gordts PLSM, Nora C, Yeang C, Witztum JL. Statin therapy increases lipoprotein(a) levels. Eur Heart J. 2020;41:2275-84.
61. Ray KK, Vallejo-Vaz AJ, Ginsberg HN, et al. Lipoprotein(a) reductions from PCSK9 inhibition and major adverse cardiovascular events: pooled analysis of alirocumab phase 3 trials. Atherosclerosis. 2019;288:194-202.

62. Burgess S, Ference BA, Staley JR, et al. Association of LPA variants with risk of coronary disease and the implications for lipoprotein(a)-lowering therapies: a mendelian randomization analysis. JAMACardiol. 2018;3:619-27.

63. Madsen CM, Kamstrup PR, Langsted A, Varbo A, Nordestgaard BG. Lipoprotein(a)-lowering by $50 \mathrm{mg} / \mathrm{dL}$ $(105 \mathrm{nmol} / \mathrm{L})$ may be needed to reduce cardiovascular disease $20 \%$ in secondary prevention: a population-based study. Arterioscler Thromb Vasc Biol. 2020;40:255-66.

64. Viney NJ, van Capelleveen JC, Geary RS, et al. Antisense oligonucleotides targeting apolipoprotein(a) in people with raised lipoprotein(a): two randomised, doubleblind, placebo-controlled, dose-ranging trials. Lancet. 2016;388:2239-53.

65. Tsimikas S, Karwatowska-Prokopczuk E, Gouni-Berthold I, etal. Lipoprotein (a) reduction in persons with cardiovascular disease. NEngl J Med. 2020;382:244-55.

66. Koren MJ, Moriarty PM, Neutel JM, et al. Safety, tolerability, and efficacy of single-dose AMG 890, a novel siRNA targeting $\operatorname{Lp}(\mathrm{a})$, in healthy subjects and subjects with elevated Lp(a). 2020. https://ir.arrowheadpharma.com/staticfiles/eeaa217f-b938-48af-ae51-03976598481d. Accessed 10 Dec 2020.

67. Afshar M, Pilote L, Dufresne L, Engert JC, Thanassoulis G. Lipoprotein(a) interactions with low-density lipoprotein cholesterol and other cardiovascular risk factors in premature acute coronary syndrome (ACS). J Am Heart Assoc. 2016;5:e3012. 